\title{
CURVAS DE CRESCIMENTO E INFLUENCIA DE FATORES NÃO-GENÉTICOS SOBRE AS TAXAS DE CRESCIMENTO DE BOVINOS DA RAÇA NELORE
}

\section{Growth curves and non-genetic factors affecting growth rate of nelore cattle}

\author{
Natascha Almeida Marques da Silva ${ }^{1}$, Luiz Henrique de Aquino $^{2}$, \\ Fabyano Fonseca e Silva ${ }^{3}$, Antonio Ilson Gomes de Oliveira ${ }^{4}$
}

\section{RESUMO}

Avaliou-se a influência de efeitos não-genéticos sobre as taxas de crescimento de bovinos da raça Nelore nas seguintes fases: do nascimento à desmama, 205 dias, (TX1); da desmama a um ano, 365 dias, (TX2); e de um ano ao sobreano, 550 dias, (TX3). Os dados constam de nove pesagens bimestrais do nascimento aos dois anos de idade, observadas entre 1978 e 1993, em 1.138 animais da raça Nelore, sendo 508 machos e 630 fêmeas. Os modelos não-lineares de Brody, Gompertz, Richards, Bertalanffy e Logístico foram ajustados aos dados de peso-idade de cada animal, para a obtenção de pesos em idades de interesse. O modelo de Gompertz foi o que melhor se ajustou aos dados e, a partir dele, foram calculadas as taxas de crescimento. Foram observadas influências $(\mathrm{P}<0,01)$ de sexo sobre TX2 e TX3, de ano sobre todas as taxas e de época de nascimento sobre TX2 e TX3. Com a identificação dos fatores não-genéticos que influenciam a eficiência do crescimento dos animais em diferentes fases de sua vida, torna-se possível otimizar as técnicas de manejo empregadas em rebanhos especializados.

Termos para indexação: Curva de crescimento, fatores não-genéticos, Nelore, taxa de crescimento.

\section{ABSTRACT}

Non-genetic effects were evaluated on growth rate of Nelore cattle from birth to 205 days (TX1), 205 to 365 days (TX2), and 365 to 550 days (TX3) of age. The data came from nine bimonthly weight-age records, from birth to two years of age, of 1.138 animals, 508 males and 630 females, collected between 1978 and 1993. Brody, Gompertz, Richards, Bertalanffy and Logistic nonlinear models were fitted for each animal, generating weight estimates at different ages. Gompertz model showed the best fitting and was chosen for growth rates computations. Effects $(\mathrm{P}<0,01)$ of sex on TX2 and TX3, year of birth on all growth rates, and season of birth on TX2 and TX3 (P<0,01), were observed. The optimization of management techniques in specialized herds is possible with the informations of the non-genetic factors that influence growth rates at distinct ages.

Index terms: Growth curve, growth rate, Nelore cattle, non-genetic factors.

(Recebido para publicação em 26 de agosto de 2002 e aprovado em 10 de dezembro de 2002)

\section{INTRODUÇÃO}

Da população bovina brasileira, estimada em cerca de 164,4 milhões de animais, $72 \%$ se concentram nas Regiões Sudeste, Sul e Centro-Oeste do País. Desses $72 \%$, aproximadamente $65 \%$ destina-se à produção de carne, obtida em regime de criação extensiva. A produção oficial de carne bovina atingiu em 2001 PIB de aproximadamente 17 bilhões de dólares, ressaltando a importância da bovinocultura de corte no cenário agropecuário nacional (ANUALPEC, 2002).
Em um sistema de produção de carne, os criadores estão cada vez mais conscientes da importância da avaliação do crescimento dos animais para melhor analisar e gerenciar a rentabilidade dessa atividade. O crescimento é uma função primordial, pois apresenta relação direta com a quantidade e a qualidade da carne, produto final da exploração. Uma das formas de se avaliar o crescimento animal é por meio de curvas de crescimento.

O aumento da massa corporal de um animal ocorre em uma seqüência temporal: pré-natal, pós-natal até a desmama, desmama até a puberdade ou sobreano

\footnotetext{
1. Acadêmica do 4o período de Zootecnia da Universidade Federal de Lavras/UFLA, Caixa Postal 37 - 37200-000 - Lavras, MG, bolsista do PIBIC/CNPq. 2. Professor Titular do Departamento de Ciências Exatas, DEX-UFLA.

3. Mestrando do Curso de Estatística e Experimentação Agropecuária, DEX-UFLA.

4. Professor Titular Aposentado da UFLA.
} 
e, para animal de reprodução, da puberdade até a maturidade, proporcionando fases com velocidades de crescimento diferentes, ou seja, em cada fase, o animal apresenta taxa de crescimento característica (GOTTSCHALL, 1999). Sob o ponto de vista econômico, a análise dessas taxas é de interesse de pesquisadores e produtores, pois seu emprego pode indicar quais as necessidades nutricionais e ambientais dos animais em cada fase de seu crescimento.

Modelos matemáticos não-lineares, desenvolvidos empiricamente para relacionar dados peso-idade, têm-se mostrado adequados para descrever curvas de crescimento (OLIVEIRA et al., 2000). Em sua maioria, apresentam três parâmetros com interpretação biológica e um quarto que se apresenta como constante matemática (ELIAS, 1998). O primeiro, peso assintótico superior (A), representa o peso na maturidade (geralmente considerado como o peso adulto). $\mathrm{O}$ segundo, definido como taxa de maturidade pós-natal (k), é um indicador da velocidade com que o animal se aproxima do peso adulto (valores altos indicam maturidade precoce e valores baixos, maturidade tardia). O terceiro, denominado de parâmetro de inflexão $(\mathrm{M})$, refere-se ao ponto em que o animal passa de uma fase de crescimento acelerada para uma fase de crescimento inibitória. $\mathrm{O}$ quarto parâmetro (b) não possui interpretação biológica, sendo utilizado para adequar o valor inicial do peso vivo, fazendo com que a curva passe pela origem (DUARTE, 1975).

Alguns requisitos devem ser seguidos para que um modelo matemático não-linear de crescimento seja descritivo de uma relação peso-idade (FITZHUGH JÚNIOR, 1976). Entre eles, destacam-se: a interpretação biológica dos parâmetros (confiabilidade), ajuste com pequenos desvios (precisão) e o grau de dificuldade do ajuste (facilidade operacional).

Segundo Alves (1986), entre outros, a forma mais prática de calcular as taxas de crescimento em uma determinada fase da vida do animal é por meio de uma aproximação discreta, ou seja, dentro de um dado intervalo de tempo desejado $\left(\mathrm{T}_{\mathrm{i}-1}\right.$ a $\left.\mathrm{T}_{\mathrm{i}}\right)$, em que os pesos correspondentes $\left(\mathrm{Y}_{\mathrm{i}-1}\right.$ e $\left.\mathrm{Y}_{\mathrm{i}}\right)$, quando não observados, são substituídos pelas suas estimativas $\left(\hat{\mathrm{Y}}_{\mathrm{i}-1}\right.$ e $\hat{\mathrm{Y}}_{\mathrm{i}}$ ), obtidas por meio do ajuste de modelos não-lineares aos dados de peso-idade.

Como todas as variáveis de produção, as taxas de crescimento também são influenciadas por fatores genéticos e outros tais, como sexo, ano e mês de nascimento (PEROTTO et al., 1997). Esses fatores não- genéticos também podem ser exemplificados, de acordo com Byers (1988), pela estação do ano, fotoperíodo, temperatura, umidade relativa, condições do curral, radiação, movimento do ar, precipitação, parasitas e a nutrição propriamente dita.

Com o estudo de taxas de crescimento por meio de estimativas de pesos ( $\hat{\mathrm{Y}}_{\mathrm{i}-1}$ e $\hat{\mathrm{Y}}_{\mathrm{i}}$ ) obtidas do ajuste de modelos não-lineares, pode-se avaliar a eficiência do crescimento animal em fases específicas, dentro da amplitude de idade utilizada nos ajustes, nas quais os dados de pesos não foram coletados. Dessa maneira, obtêm-se informações importantes que podem ser utilizadas para indicar a demanda nutricional nessas fases e também para elaboração de programas de melhoramento genético. $\mathrm{O}$ estudo dos fatores não-genéticos que influenciam o crescimento desses animais torna-se importante para se determinar práticas de manejo adequadas e, também, para obter estimativas de valor genético com maior precisão.

Dessa forma, objetiva-se com o presente estudo definir o modelo não-linear que melhor se ajusta ao crescimento de bovinos Nelore, obter estimativas de pesos a partir desse modelo, que serão utilizadas no cálculo de taxas de crescimento, e identificar fatores nãogenéticos que influenciam essas taxas.

\section{MATERIAL E MÉTODOS}

Os dados utilizados neste trabalho foram fornecidos pela ABCZ (Associação Brasileira de Criadores de Zebu), localizada em Uberaba, Minas Gerais. Eles constam de nove pesagens, aproximadamente bimestrais, uma vez que elas não se realizavam exatamente a cada sessenta dias, do nascimento aos dois anos de idade de 1.138 animais da raça Nelore, 508 machos inteiros e 630 fêmeas, pertencentes a um mesmo rebanho localizado na cidade de Araguari, Minas Gerais. Foram considerados somente dados relativos a animais criados exclusivamente em pastagens e filhos de vacas com idade entre três e dez anos. As épocas de nascimento estudadas foram: seca (abril a setembro) e chuvosa (outubro a março), abrangendo o período de 1978 a 1993.

As curvas de crescimento médias foram estimadas a partir das médias das idades, em dias, e dos pesos dos animais a cada pesagem, e as curvas individuais, estimadas por meio das observações de peso-idade de cada animal. Os cinco modelos de crescimento ajustados aos dados de peso-idade apresentam as seguintes expressões matemáticas incluídas na Tabela 1. 
Os ajustes dos modelos de crescimento foram realizados por meio do procedimento MODEL do programa Statistical Analysis Systems (SAS INSTITUTE, 1995). Os valores iniciais para estabelecer a convergência dos dados foram fornecidos pela literatura especializada (TEDESCHI, 1996; ELIAS, 1998). Os valores iniciais para a convergência das curvas individuais foram as estimativas dos parâmetros geradas pelo ajuste da curva média.

Para indicar o modelo que melhor se ajustou aos dados, foram considerados os seguintes avaliadores de qualidade, conforme sugeridos por Draper e Smith (1998): porcentagem de convergência, coeficiente de determinação ajustado para o número de parâmetros do modelo $\left(\mathrm{R}_{\mathrm{a}}^{2}\right)$, autocorrelação residual de primeira or$\operatorname{dem}(r)$ e estatística de Durbin e Watson (DW).

A partir da definição do melhor modelo, ou seja, o que melhor descreveu o crescimento dos animais, estimaram-se os pesos para cada animal $i$ $\left(\hat{Y}_{i(j)}\right.$ e $\left.\hat{Y}_{i(k)}\right)$ utilizados para calcular as taxas de crescimento, respectivamente, nas idades $j$ e $k$. Essas taxas foram estimadas para as seguintes fases de crescimento: do nascimento à desmama (205 dias), TX1; da desmama a um ano (365 dias), TX2; e de um ano ao sobreano (550 dias), TX3. As fórmulas para a taxa de crescimento, de acordo com Alves (1986), são dadas por:

$$
\begin{gathered}
T X 1=\frac{\hat{Y}_{205}-\hat{Y}_{0}}{205-0} ; \quad T X 2=\frac{\hat{Y}_{365}-\hat{Y}_{205}}{365-205} ; \\
T X 3=\frac{\hat{Y}_{550}-\hat{Y}_{365}}{550-365} ;
\end{gathered}
$$

A influência de fatores não-genéticos sobre as taxas de crescimento nas diferentes fases da vida do animal foi avaliada por meio do modelo contendo efeitos fixos de sexo, ano e época de nascimento e idade da vaca ao parto. Esse último foi considerado como uma covariável, estudada nas formas linear e quadrática. A análise de variância envolvendo as variáveis desse modelo foi realizada por meio do procedimento GLM (SAS INSTITUTE, 1996).

\section{RESULTADOS E DISCUSSÃO}

O ajuste individual dos modelos não-lineares de crescimento aos dados de peso-idade dos animais gerou estimativas para os parâmetros, as quais são apresentadas na Tabela 2, na qual se vê que as funções de Brody e Richards superestimaram o peso assintótico, concordando com os resultados de Elias (1998), ao passo que as demais (Gompertz, Logística e Bertalanffy) apresentaram valores próximos aos encontrados na literatura especializada (TEDESCHI, 1996). A média dos pesos à última pesagem (2 anos) de todos os modelos ajustados: Brody $(315,96)$, Gompertz (310,33), Logístico $(306,96)$, Richards $(319,02)$ e Bertalanffy $(311,90)$ apresentaram-se dentro da amplitude estipulada na literatura especializada (TEDESCHI, 1996), portanto, até nessa idade, esses modelos descrevem bem o crescimento dos animais. A grande amplitude de variação observada para o parâmetro k, de 0,002 a 0,018, deve-se à alta correlação negativa (DUARTE, 1976; TEDESCHI, 1996) desse parâmetro com o peso adulto (A), ou seja, as funções de Brody e Richards não estimaram bem o peso assintótico.

Constata- se pela Tabela 3 que a porcentagem de convergência foi maior para a função de Gompertz e menor para a função de Richards. Segundo Elias (1998), a maior dificuldade de ajuste para a função de Richards é em razão da variabilidade do seu ponto de inflexão. As funções de Brody e de Gompertz apresentaram valores mais altos para o $\mathrm{R}_{\mathbf{a}}^{2}$, indicando maior aderência, se comparadas com as demais funções.

\begin{tabular}{|c|c|}
\hline Modelos não-lineares de crescimento & Equação \\
\hline Gompertz (LAIRD, 1965) & $Y=A \exp \left(-b \exp ^{-K t}\right)$ \\
\hline Brody (BRODY, 1945) & $Y=A\left(1-b \exp ^{-K t}\right)$ \\
\hline Logístico (NELDER, 1961) & $\mathrm{Y}=\mathrm{A}\left(1+\mathrm{b} \exp ^{-\mathrm{Kt}}\right)^{-1}$ \\
\hline Bertalanffy (BERTALANFFY, 1957) & $Y=A\left(1-b \exp ^{-K t}\right)^{3}$ \\
\hline Richards (RICHARDS, 1959) & $\mathrm{Y}=\mathrm{A}\left(1-\mathrm{b} \exp ^{-\mathrm{Kt}}\right)^{\mathrm{M}}$ \\
\hline
\end{tabular}

TABELA 1 - Modelos não-lineares de crescimento e suas respectivas equações. 
Em relação à avaliação de autocorrelação residual, todas as funções apresentaram comportamentos similares, pois os valores da estatística DW não foram significativos e $r$ se apresenta próximo a zero, indicando a ausência de autocorrelação residual (HOFFMAN e VIEIRA, 1998). Essa ausência também foi confirmada por meio de análises de resíduos.

Sob o ponto de vista prático, a função de Gompertz é a mais indicada para descrever o crescimento de gado Nelore, pois, além de apresentar bom ajuste, a maior porcentagem de convergência confere maior segurança para experimentos que envolvem as estimativas dos parâmetros $\mathrm{A}, \mathrm{b}, \mathrm{e} \mathrm{k}$. Quanto à porcentagem de convergência, observa-se que a amplitude de 36,99 (Richards) a 67,48 (Gompertz) encontra-se dentro da faixa de valores apresentados por Mazzini (2001), os quais variam de 23,13 (Brody) a 100 (Logística). Essa grande variabilidade de convergência deve-se às características dos dados, como intervalo entre pesagens e número de pesagens; quanto a isso, Tedeschi (1996) comenta que baixos percentuais são obtidos geralmente quando se utilizam animais jovens para a estimação de peso adulto. Contudo, tendo em vista o baixo percentual de convergência para a maioria dos modelos, o que limita o número de dados utilizados na análise, talvez seria mais interessante utilizar os ganhos de peso relativos entre as idades observadas. Desse modo, o estudo ficaria restrito a esses intervalos de idade, não sendo possível, assim, estudar taxas de crescimento em intervalos de interesse específicos (KAPS et al., 2000), como é o caso do presente estudo.

Nota-se pela Figura 1, na qual se vê o gráfico do ajuste médio das funções de Gompertz e Brody, que do nascimento aos dois anos de idade o comportamento dessas funções é bem parecido, indicando que nesse período da vida do animal ambas podem ser utilizadas para representar o crescimento médio dos animais

TABELA 2 - Médias (erros-padrão) das estimativas individuais para o peso assintótico (A) em kg, constante de integração (b), taxa de maturidade (k) em (dias) ${ }^{-1}$ e parâmetro de inflexão (M).

\begin{tabular}{lcccc}
\hline \multicolumn{1}{c}{ Funções } & $\mathbf{A}(\mathbf{E P})$ & $\mathbf{b}(\mathbf{E P})$ & $\mathbf{k}(\mathbf{E P})$ & $\mathbf{M}(\mathbf{E P})$ \\
\hline Brody & $428,73(7,23)$ & $0,91(0,0012)$ & $0,002(0,00004)$ & - \\
Gompertz & $378,68(6,03)$ & $2,14(0,0118)$ & $0,007(0,00344)$ & - \\
Logística & $358,88(5,56)$ & $5,59(0,0566)$ & $0,010(0,03401)$ & - \\
Richards & $474,87(13,04)$ & $0,71(0,0167)$ & $0,002(0,00010)$ & $5,01(0,44)$ \\
Bertalanffy & $388,75(6,40)$ & $0,51(0,0023)$ & $0,018(0,01516)$ & - \\
\hline
\end{tabular}

TABELA 3 - Avaliadores de qualidade dos ajustes individuais representados pela \% de convergência, coeficiente de determinação ajustado $\left(\mathrm{R}_{\mathbf{a}}^{\mathbf{2}}\right)$, autocorrelação residual de primeira ordem $(r)$ e estatística de Durbin e Watson (DW).

\begin{tabular}{lcccc}
\hline \multicolumn{1}{c}{ Funções } & \% convergência & $\mathbf{R}_{\mathbf{a}}^{\mathbf{2}}$ & $\boldsymbol{r}$ & DW \\
\hline Brody & 56,85 & 0,958 & $-0,118$ & 1,83 \\
Gompertz & 67,48 & 0,931 & $-0,068$ & 1,57 \\
Logística & 65,81 & 0,901 & 0,012 & 1,33 \\
Richards & 36,99 & 0,908 & $-0,141$ & 1,83 \\
Bertalanffy & 65,81 & 0,912 & $-0,077$ & 1,64 \\
\hline
\end{tabular}

Ciênc. agrotec., Lavras, v. 28, n. 3, p. 647-654, maio/jun., 2004 
As médias das taxas de crescimento $(\mathrm{kg} / \mathrm{dia})$ nas diferentes fases da vida do animal, foram 0,4641 (TX1), 0,4370 (TX2) e 0,3104 (TX3), indicando que, com o decorrer da idade, os animais crescem com menor velocidade, concordando com Gottschall (1999). As estimativas dessas taxas foram submetidas à análise de variância, sendo os resultados apresentados na Tabela 4 .

Pelos resultados, evidencia-se o efeito significativo de sexo sobre TX2 e TX3 $(\mathrm{P}<0,01)$, indicando que após a desmama os machos crescem com maior velocidade em relação às fêmeas (Figura 2), concordando com Gottschall (1999). Segundo esse autor, machos inteiros apresentam maior velocidade de crescimento que as fêmeas; porém, o mesmo não se verifica para machos castrados. Em relação ao efeito não-significativo de sexo sobre TX1, Machado et al. (1997) ressaltam que a superioridade dos machos sobre as fêmeas em relação ao ganho de peso tende a aumentar com a idade; por isso no presente estudo, essa superioridade só foi constatada a partir da desmama.

$\mathrm{O}$ efeito $(\mathrm{P}<0,01)$ de ano de nascimento sobre todas as taxas pode ser explicado pelo aperfeiçoamento das práticas de manejo no decorrer dos anos estudados, refletindo, assim, em tendência crescente (Figura 3) das taxas de crescimento. Esse resultado concorda com o estudo de Perotto et al. (1997), os quais também observaram alta significância para o ano de nascimento $(\mathrm{P}<0,001)$.

A influência de época de nascimento sobre TX2 e TX3 $(\mathrm{P}<0,01)$ revela que animais jovens (desmamados) e adultos que nasceram no período seco cresceram mais rapidamente (Figura 4) do que os que nasceram no período chuvoso, pois, na região de Araguari, Triângulo Mineiro, além da menor incidência de doenças na época seca, os animais, quando desmamados, encontram maior disponibilidade de pastagens.

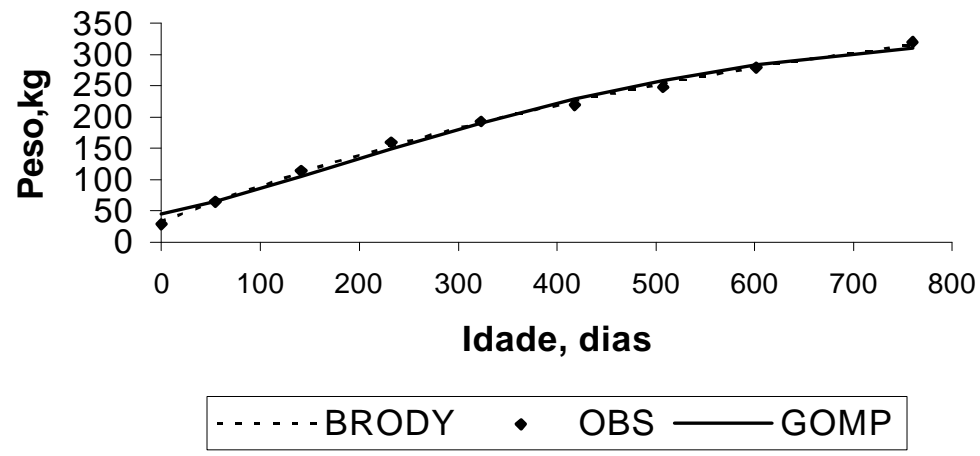

FIGURA 1 - Ajuste das Funções de Crescimento de Gompertz e de Brody aos dados de peso-idade de Nelore.

TABELA 4 - Quadrados Médios dos efeitos de sexo, ano, época de nascimento e idade da vaca sobre as taxas de crescimento de gado Nelore.

\begin{tabular}{|c|c|c|c|c|}
\hline \multirow[t]{2}{*}{ Fontes de Variação } & \multirow[t]{2}{*}{ Graus de liberdade } & \multicolumn{3}{|c|}{ Quadrados Médios } \\
\hline & & TX1 ${ }^{1}$ & $\mathrm{TX2}^{2}$ & $\mathbf{T X 3}^{\mathbf{3}}$ \\
\hline Sexo & 1 & 0,0350 & $0,9699 * *$ & $2,4248 * *$ \\
\hline Ano & 12 & $0,2643 * *$ & $0,3056^{* *}$ & $0,1928 * *$ \\
\hline Época & 1 & 0,0101 & $0,1178 * *$ & $0,1191 * *$ \\
\hline Idade da vaca (Linear) & 1 & 0,0126 & 0,0202 & $0,0675^{*}$ \\
\hline Idade da vaca (Quadrática) & 1 & 0,0042 & 0,0275 & $0,0669 *$ \\
\hline Erro & 751 & 0,0158 & 0,0110 & 0,0133 \\
\hline \multicolumn{5}{|c|}{$\begin{array}{l}{ }_{1}^{1} \text { Taxa de crescimento do nascimento a desmama ( } 205 \text { dias) } \\
{ }^{2} \text { Taxa de crescimento da desmama a um ano (365 dias) } \\
{ }^{3} \text { Taxa de crescimento de um ano ao sobreano (550 dias) } \\
* \mathbf{P}<0,05 ; * * \mathbf{P}<0,01\end{array}$} \\
\hline
\end{tabular}


A idade da vaca ao parto, estudada como covariável nas formas linear e quadrática, afetou somente TX3 $(\mathrm{P}<0.05)$, resultado que discorda da literatura (PAZ, 1997), pois, no período de maior dependência do animal em relação à mãe (TX1), esse efeito não se mostrou significativo; portanto, não haveria de influenciar TX3. Diante desse resultado, torna-se necessária a representação gráfica desse efeito, assim como a equação de regressão gerada e o coeficiente de determinação $\left(\mathrm{R}^{2}\right)$, os quais são mostrados na Figura 5.
A não-influência de época sobre TX1 e de idade da vaca ao parto sobre TX1 e TX2 pode estar relacionada às condições edafoclimáticas específicas da região, como, por exemplo, a estacionalidade na produção de alimentos em termos qualitativos e quantitativos (SARMENTO et al., 2003), que contribuíram direta ou indiretamente sobre a condição corporal da vaca, refletindo, assim, sobre ganho de peso dos bezerros.

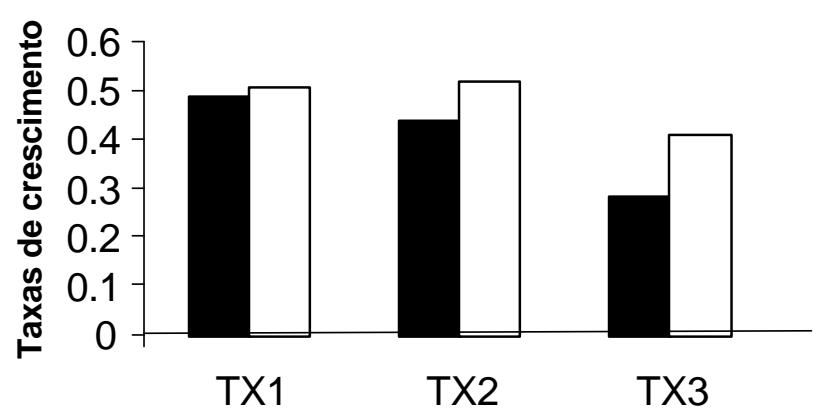

Fêmea $\square$ Macho

FIGURA 2 - Efeito do sexo sobre as taxas de crescimento de gado Nelore.
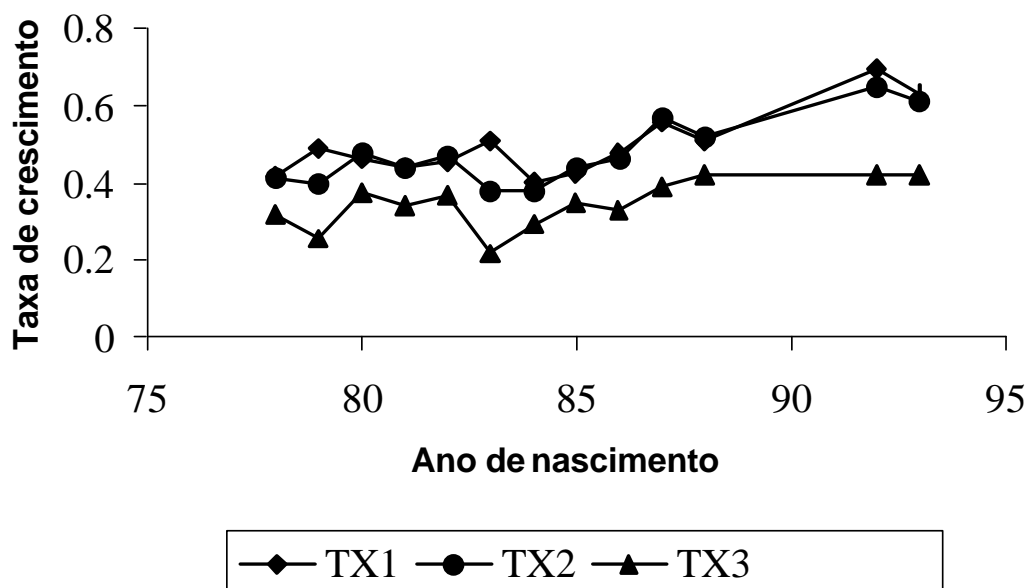

FIGURA 3 - Efeito do ano de nascimento sobre as taxas de crescimento de gado Nelore. 


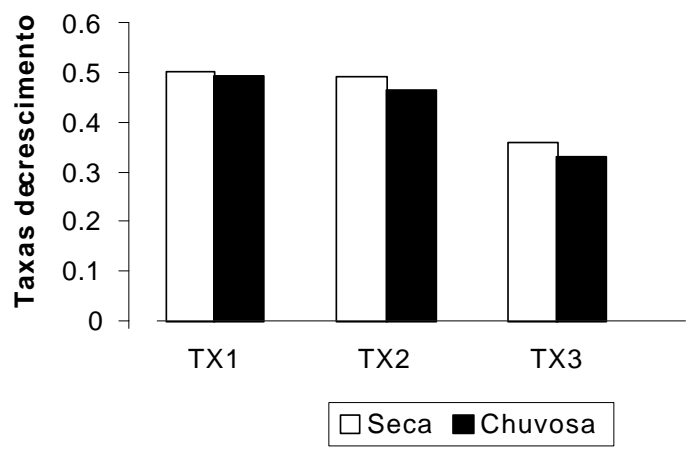

FIGURA 4 - Efeito de época de nascimento sobre as taxas de crescimento de gado Nelore.

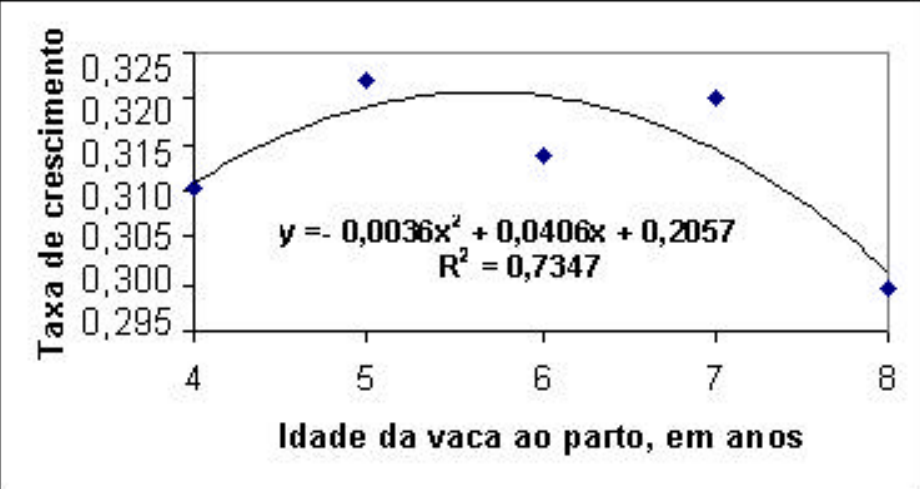

FIGURA 5 - Efeito da idade da vaca sobre TX3 (entre um ano e 550 dias).

\section{CONCLUSÕES}

A função de Gompertz foi escolhida para descrever o crescimento de bovinos Nelore por apresentar bom ajuste, maior porcentagem de convergência e estimativas com interpretação biológica.

Os efeitos de sexo, ano e época de nascimento são importantes fatores não-genéticos que influenciam a eficiência (taxas) do crescimento de bezerros Nelore, em especial o crescimento pós-desmame.

\section{REFERÊNCIAS BIBLIOGRÁFICAS}

ALVES, R. G. O. Estudo genético de características reprodutivas em suínos e avaliação de curvas de crescimento em cruzamentos dialélicos. 1986. $124 \mathrm{f}$. Tese (Doutorado em Zootecnia) - Universidade Federal de Viçosa, Viçosa, 1986.
ANUALPEC. Anuário da Pecuária Brasileira. São Paulo, 2002. 104 p.

BERTALANFFY, L. von. Quantitative laws in metabolism and growth. Quarterly Review of Biology, Chicago, v. 32, p. 217-230, 1957.

BRODY, S. Bioenergetics and growth. New York: Reinhold, 1945. 1023 p.

BYERS, F. M. Nutrition in growth: the ruminant animal digestive physiology and nutrition. New Jersey: Reston Book, 1988. 447 p.

DRAPER, N. D.; SMITH, H. Applied regression analysis. New York: John Wiley \& Sons, 1998. $706 \mathrm{p}$. 
DUARTE, F. A. M. Estudo da curva de crescimento de animais da raça Nelore (Bos taurus indicus) através de cinco modelos estocásticos. 1975. 284 f. Tese (Livre-Docência) - Faculdade de Medicina de Ribeirão Preto, Universidade de São Paulo, Ribeirão Preto, 1975.

ELIAS, M. A. Análise de curvas de crescimento de vacas das raças Nelore, Guzerá e Gir. 1998. 128 f. Dissertação (Mestrado em Zootecnia) - Escola Superior de Agricultura de "Luiz de Queiroz”, Piracicaba, 1998.

FITZHUGH JÚNIOR, H. A. Analysis of growth curves and strategies for altering their shapes. Journal of Animal Science, Champaign, v. 42, n. 4, p. 10361051, 1976.

GOTTSCHALL, C. S. Impacto nutricional na produção de carne e curva de crescimento. In: LOBATO, J. F. P.; BARCELLOS, J. O. J.; KESSLER, A. M. Produção de bovinos de corte. Porto Alegre: EDIPUCRS, 1999. p. 169-192.

HOFFMAN, R.; VIERA, S. Análise de regressão: uma introdução à econometria. 3. ed. São Paulo: HUCITEC, 1998. 397 p.

KAPS, M.; HERRING, W. O.; LAMBERSON, W. R. Genetic and environmental parameters for traits derived from the Brody growth curve and their relationships with weaning weigth in Angus cattle. Journal Animal Science, Champaign, v. 78, p. 14361442,2000

LAIRD, A. K. Dynamics of relative growth. Growth, Bar Harbor, v. 29, p. 249-263, 1965.

MACHADO, P. F. A.; AQUINO, L. H.; GONÇALVES, T. M. Influência de fatores de meio sobre os pesos ao nascer, 205 dias, 365 dias e 550 dias de idade de animais da raça nelore, no estado de Minas Gerais. Ciência e Agrotecnologia, Lavras, v. 21, n. 3, p. 373385, jul./set. 1997.

MAZZINI, A. R. A. Análise da curva de crescimento de machos Hereford considerando heterogeneidade de variâncias e autocorrelação dos erros. 2001. 94 p. Dissertação (Mestrado) - Universidade Federal de Lavras, Lavras, 2001.
NELDER, J. A. The fitting of a generalization of the logistic curve. Biometrics, Washington, v. 17, p. 89100, 1961.

OLIVEIRA, H. N.; LÔBO, R. B.; PEREIRA, C. S. Comparação de modelos não-lineares para descrever o crescimento de fêmeas da raça Guzerá. Pesquisa Agropecuária Brasileira, Brasília, v. 35, n. 9, p. 1843-1851, set. 2000.

PAZ, C. C. P. de. Efeitos ambientais e genéticos que afetam o ganho de peso pré-desmame em bovinos da raça Nelore. 1997. 117 f. Dissertação (Mestrado em Zootecnia) - Universidade Estadual Paulista, Jaboticabal.

PEROTTO, D.; CASTANHO, M. J. de P.; CUBAS, A. C.; ROCHA, J. L.; PINTO, J. M. Efeitos genéticos sobre as estimativas dos parâmetros das curvas de crescimento de fêmeas bovinas Gir, Guzerá, Holandês x Gir e Holandês x Guzerá. Revista Brasileira de Zootecnia, Viçosa, v. 26, n. 4, p. 719-725, 1997.

RICHARDS, F. J. A flexible growth function for empirical use. Journal of Experimental Botany, Oxford, v. 10, p. 290-300, 1959.

SARMENTO, J. L. R.; PEREIRA FILHO, E. C.; RIBEIRO, M. A.; MAGALHÃES FILHO, R. Efeitos ambientais e genéticos sobre o ganho em peso diário de bovinos nelore no estado da Paraíba. Revista Brasileira de Zootecnia, v. 32, n. 2, p. 325-330, 2003.

STATISTICAL ANALYSIS SYSTEM INSTITUTE. User's guide: version 6. 2. ed. Cary, 1995.

STATISTICAL ANALYSIS SYSTEM INSTITUTE. User's guide: statistics version 6. 4. ed. Cary, 1996. $168 \mathrm{p}$.

TEDESCHI, L. O. Determinação dos parâmetros da curva de crescimento de animais da raça Guzerá e seus cruzamentos alimentados a pasto, com e sem suplementação. 1996. 140 f. Dissertação (Mestrado em Zootecnia) - Escola Superior de Agricultura de "Luiz de Queiroz", Piracicaba, 1996. 